DOI: $10.25100 /$ pfilosofica.v0i50.8783

\title{
EL ENFOQUE INTENCIONAL EN LAS CIENCIAS SOCIALES: UNA MIRADA ESTRUCTURALISTA DE LAS TEORÍAS CIENTÍFICAS INTENCIONALES
}

$\left\{\begin{array}{r}\text { Juan Manuel Jaramillo Uribe } \\ \text { Facultad de Humanidades, Universidad del Valle, Cali, Colombia }\end{array}\right]$

\begin{abstract}
Resumen
Frente a los distintos modelos de explicación en las ciencias (nomológicodeductivo, genético, teleológico, disposicional, etc.) la explicación intencional tiene especial relevancia en las ciencias sociales y en la explicación/predicción del comportamiento de algunas entidades artefactuales. Dicha explicación permite diferenciar las ciencias sociales de las naturales. En este escrito se propone presentar, en el contexto de la concepción del estructuralismo metateórico, dicha explicación intencional y presentar algunos casos de su aplicación en teorías como la teoría de elección racional, la teoría de juegos y la teoría de decisiones cuando tales teorías se aplican en las ciencias sociales, en especial, en economía.
\end{abstract}

Palabras clave: ciencias naturales y sociales; intencionalidad; teorías intencionales; explicación intencional; estructuralismo metateórico.

Cómo citar este artículo: Jaramillo-Uribe, J.M. (2020). El enfoque intencional en las ciencias sociales: Una mirada estructuralista de las teorías científicas intencionales. Praxis Filosífica, (50), 141-160. doi: 10.25100/pfilosofica.v0i50.8783

Recibido: 14 de agosto de 2019. Aprobado: 14 de septiembre de 2019. 


\title{
The Intentional Approach in Social Sciences: A Structuralist View of Intentional Scientific Theories
}

\author{
Juan Manuel Jaramillo Uribe ${ }^{1}$
}

\begin{abstract}
With respect to the different models of explanation in the sciences (nomological-deductive, genetic, teleological, dispositiona, etc.) the intentional explantion has special relevance in the social siences and in the explanation allows to differentiate the social sciences from the natural ones. In this paper it si proposed to present, in the context of the metatheoretical structualist view, this intentional explanation and present some cases of its applications in theories such as the Theory of rational choise, games theory and decision theory when such theories are applied in the social sciences, especiallly in economics.
\end{abstract}

Keywords: Natural and social sciences; Intentionality; Intentional Theories; Metatheoretical Structuralism.

${ }^{1}$ Doctor en Filosofía de la Universidad de Antioquia bajo la dirección del Dr. C. Ulises Moulines (U. de Munich). Jubilado de la U. Del Valle donde fue profesor, Jefe de Departamento de Filosofía, Secretario General y Rector (E) en varias oportunidades. Desde el 2000 miembro del grupo de investigación internacional "Reconstrucciones históricas y reconstrucciones racionales" con sede en Buenos Aires. Los intereses filosóficos se centran en temas ligados al Idealismo alemán (en especial Kant), la filosofía analítica, la semántica filosófica y la filosofía de la ciencia (en especial el programa meta-teórico estructuralista de reconstrucción de teorías). Entre sus publicaciones es autor de Es la ciencia una rama de la literatura fantástica. Pretexto para una reflexión sobre el realismo (2001); Teoría intuitiva de conjuntos (2005); Filosofía de la tecnología (2008) y co-autor de Thomas Kuhn (1999); El camino desde Kuhn. La inconmensurabilidad hoy (2012); La filosofía de la ciencia en Iberoamérica: Metateoría Estructural (2012); Monográfico de Ágora: Nuevas contribuciones iberoamericanas a la metateoría estructuralista (2012); Monográfico de Perspectivas: Dossiê filosofia da ciência no século xxi - contribuições da metateoria estruturalista (2018); Exploraciones pluralistas. Las filosofías de C. Ulises Moulines (en prensa). Además de numerosos artículos en revistas nacionales e internacionales.

ORCID: 0000-0002-8156-2333 E-mail: jaramillo.juanmanuel@gmail.com 


\title{
EL ENFOQUE INTENCIONAL EN LAS CIENCIAS SOCIALES: UNA MIRADA ESTRUCTURALISTA DE LAS TEORÍAS CIENTÍFICAS INTENCIONALES
}

\author{
Juan Manuel Jaramillo Uribe \\ Facultad de Humanidades, Universidad del Valle, Cali, Colombia
}

\section{Introducción}

La explicación/predicción es el objetivo central teórico de la ciencia entendida como un conjunto de teorías. Sin embargo, dado el cúmulo de debates que se han producido en torno a la explicación científica y a los diferentes tipos de teorías sobre ella, resultaría imposible establecer una única estructura subyacente de explicación/predicción para todas las teorías científicas. Esto, sin embargo, no excluye la posibilidad de proponer algún tipo de modelo formal de explicación/predicción que, sin pretender una validez absoluta y universal, tenga un dominio de aplicaciones más o menos amplio, tanto en el campo de las ciencias naturales, como en el de las ciencias sociales y humanas.

Desde finales del s. XIX se planteó una escisión entre las llamadas "ciencias del espiritu" ["Geisteswissenchaften"] y las llamadas "ciencias de la naturaleza" ["Naturwissenchaften"] que, de algún modo, se corresponde con la distinción más contemporánea entre ciencias sociales y naturales ${ }^{2}$. En 1971, von Wright distingue dos tradiciones: la aristotélica y la galileana. A ellas corresponden dos objetivos metodológicos distintos: la comprensión [Verstehen] y la explicación [Erklärung,], respectivamente, habitualmente caracterizadas en términos de explicación teleológica versus explicación

\footnotetext{
${ }^{2}$ En Alemania las "ciencias del espíritu” comprendían disciplinas tales como la retórica, la poética, la ética, la filosofía del derecho, la economía política, la sociología, la hermenéutica y el estudio de las religiones que viene a coalescencia con la historia en razón de sus temas. A estas ciencias se las llamaba en Francia "ciencias morales y políticas".
} 
causal, si bien, hay que decirlo, la explicación teleológica es una forma de explicación causal.

Quienes defienden la tradición aristotélica, apuntan a la comprensión [Verstehen] de la conducta social mediante el establecimiento de las razones (creencias, deseos, intenciones, etc.) que llevaron a los agentes a actuar. Para Davidson (1980) tales las razones, en tanto representaciones mentales (cerebrales), fungen como causas de la acción, pues las metas carecen de eficacia causal. De este modo - como lo observa Bunge (1996) - "la diferencia entre las explicaciones por causas (eficientes) y por razones (suficientes) se reduce a la distinción entre causas externas e internas" ( $\mathrm{p}$. 200).

Von Wright defensor de la primera tradición, destaca en las ciencias sociales la "dimensión semántica de la intencionalidad" como su rasgo más característico, algo que las distingue de las ciencias naturales. En tal sentido escribe:

La comprensión [Verstehen] se encuentra [...] vinculada a la intencionalidad, de una manera en que la explicación no lo está. Se comprenden los objetivos y propósitos de un agente, el significado de un símbolo o de un signo, el sentido de una institución social o de un rito religioso (1979, p. 24).

En el otro bando, quienes defienden la tradición galileana apuntan a un tipo general de explicación/predicción de los fenómenos mediante su subsunción bajo leyes, en términos del que se conoce como "modelo de cobertura legal inferencial" que comprende un modelo nomológicodeductivo particular (NDP) o de hechos particulares y un modelo nomológicodeductivo general (NDG) o hechos generales, $v$. gr., la explicación de leyes derivándolas de otras ${ }^{3}$. En estos modelos se habla de leyes que describen regularidades estrictamente generales y de leyes estadístico-probabilistas.

Sin embargo, desde 1943 Rosenblueth, Wiener y Bigelow en su escrito "Behavior, Purpose, and Teleology" se había planteado la posibilidad de extender ese modelo a las ciencias de la conducta, pudiéndose hablar de "conducta teleológica", no solo respecto del comportamiento de los organismos vivos, sino también de todos los sistemas autorregulados $\mathrm{u}$ homeostáticos.

\footnotetext{
${ }^{3}$ En Hempel-Oppenheim (1948) se presenta el núcleo básico de este modelo, aunque Hempel lo desarrolla en los 60's del siglo pasado, sin dejar de reconocer que sus orígenes se encuentran en Aristóteles, Mill, Campbell y Popper.
} 
En el caso de los organismos vivos los biólogos se ocupan de estudiar las funciones de los órganos y procesos vitales mediante un "análisis teleológico", al considerar que se trata de procesos dirigidos a un fin, como lo señaló Nagel (1961). En su opinión tales análisis marcan la frontera entre la biología y las ciencias físicas ${ }^{4}$. Su forma general dice: "La función $A$ de un sistema $S$ con una organización [diseño] $D$ es permitir a $S$, en un medio [entorno] E realizar el proceso P " (p. 367). La clorofila, por ejemplo, otorga color verde en las plantas y su función es permitir que estas realicen el proceso de fotosíntesis consistente en la transformación de la energía lumínica en energía química para así garantizar los nutrientes necesarios para la supervivencia de dicha especie.

El asunto es que la postura teleológica de Nagel (1961), a pesar de su posición marcadamente neopositivista, entraña una metafísica oculta en la que se confunde la supuesta "explicación teleológica" con una explicación funcional donde se tendrían en cuenta las funciones individuales de los elementos de un sistema biológico, para engendrar una función global determinada.

Fue Darwin, quien a través de su modelo de evolución por selección natural rechazó desde mediados del siglo XIX la explicación teleológica, a menos que apelemos a la metáfora del diseño y pensemos los organismos o sus partes como artefactos producto de la evolución, como dice Ruse (2000). Es importante advertir que las explicaciones biológicas no suponen la existencia de ningún agente sobrenatural como en el caso de la "teoría" del diseño inteligente. Lo que ofreció Darwin con su explicación filogenética, fue una explicación puramente naturalista del 'diseño biológico' producto de la evolución, eliminando así la teleología de la biología, como lo advierte González (2016). Kuhn (2002) destaca lo anterior cuando al explicar la evolución de las teorías científicas mediante la teoría de la evolución darwiniana recuerda que se trata de un procesos "dirigido desde atrás, no atraído hacia adelante delante -como la evolución desde, en lugar de la evolución hacia-." (pp. 120-121). No obstante, lo anterior continúa siendo un debate central en la biología y a pesar de los esfuerzos de los biólogos por expulsar la teleología de sus explicaciones, hay que decir que el lenguaje teleológico o su forma disfrazada, teleonómico (Mayr, 2006), aún sigue presente en esta disciplina, algo a lo que ni el mismo Darwin pudo escapar.

\footnotetext{
${ }^{4}$ Jon Elster distingue las explicaciones causales, de las explicaciones funcionales y de las explicaciones intencionales y señala que la física es el paradigma o "modelo estándar" de las explicaciones causales, la biología de las funcionales y las ciencias sociales de las intencionales, sin con ello rechazar la posibilidad de que se puedan hacer explicaciones intencionales en biología o causales en biología y en ciencias sociales (Elster, 1995).
} 
Abundante literatura existe en torno a las tradiciones aristotélica y galileana en el debate ciencias naturales/ciencias sociales y no es el propósito en este escrito detenerme en ellas. Sostendré que en las ciencias sociales y humanas, la explicación/predicción no es algo ajeno. Lo que, me propongo es presentar el modelo de explicación científica que propone el estructuralismo metateórico (en adelante $E M$ ) y que básicamente consiste en insertar o subsumir un modelo de datos (en adelante $\mathrm{M}_{\mathrm{D}}$ ) en un modelo teórico ${ }^{5}$. En tal modelo, algunos de sus términos/conceptos T-teóricos (de conformidad con el criterio sneedeano de T-teoricidad) indispensables para realizar explicaciones/predicciones científicas, pues son ellas las responsables explicativo/predictivas del comportamiento de las parcelas de la realidad expresadas mediante modelos de datos $\mathrm{M}_{\mathrm{D}}$, pueden recibir una interpretación causal, en cuyo caso esta explicación por subsunción modelo-teórica sería una explicación causal. Dicho modelo sería el mismo para las ciencias naturales y sociales, solo que en estas últimas el componente T-teórico incluye términos/conceptos intencionales que es necesario introducir a efectos de la explicación/predicción.

\section{Los sistemas y las explicaciones intencionales}

John Searle, en "Mentes, Cerebros y Programas" ["Minds, Brains and Programs"] (1980), distingue dos variantes relevantes de la intencionalidad: (i) la intencionalidad intrínseca que se refiere a nuestros pensamientos, deseos, intenciones en el sentido corriente del término y, (ii) la intencionalidad derivada que es la que adscribimos a sistemas distintos de nosotros como artefactos, programas de computación, robots, etc. Para Dennett (2000) estos objetos

tienen intencionalidad [derivada] solo por la cortesía de la especie de préstamo generoso que les hace nuestra propia mente. La intencionalidad derivada de nuestras representaciones artefácticas es parásita de la intencionalidad genuina, intrínseca y originaria que se esconde tras su creación (p. $66)$.

En esta clase de intencionalidad los explananda de las $\mathrm{T}_{\mathrm{I}}$ no solo serían personas o más específicamente sus conductas, sino también los animales, las plantas y algunos artefactos complejos. A todos ellos Dennett los denominan

${ }^{5}$ Como lo ha reconocido uno de los más importantes representantes del EM, C.U. Moulines (2007), este modelo de explicación por subsunción modelo-teórica sintetiza dos enfoques, el unificacionista de Friedman y Kitcher y el causalista de W. Salmon, modificado y enriquecido con los aportes de Bartelbort (1966), Forge (1985) y Díez (1998) (En Moulines, 2007, p. 8). 
sistemas intencionales, y a su explicación/predicción: explicación/predicción intencional.

Pero el sentido originario y restringido de la noción de "sistema intencional" refiere únicamente a personas en tanto agentes racionales en los que la elección de sus acciones se basa en creencias, deseos, preferencias, juicios, sentimientos, temores, hábitos, etc., i.e., en estados intencionales en los que la intencionalidad -del latín tendere como "dirigido a"- constituye el sello distintivo de lo mental (Tercera tesis de Brentano).

Refiriéndose a este sentido originario, Searle (1983) ve necesario diferenciar en el análisis lingüístico de la conducta intencional tres componentes básicos: los agentes intencionales, las actitudes intencionales $y$ los contenidos intencionales. En una frase como " $x$ cree que ' $p$ ", " $x$ " es el agente intencional, "cree que" la actitud intencional" $\mathrm{y}$ " " $p$ " (entre comillas) la proposición que expresa el contenido intencional. En este caso, se trata de una subclase especial de agentes intencionales que, poseen un lenguaje articulado, se comunican con otros y son el marco para una teoría de la conciencia ${ }^{6}$.

Sin embargo, las acciones intencionales del agente intencional (que no hay que confundir con el sistema intencional como lo suele hacer Dennett) se encuentran inmersas en un contexto social y, en ocasiones, se realizan como acciones colectivas, aunque para Searle (1983; 1990; 1995; 2001; 2010) dicha intencionalidad colectiva no es la simple sumatoria de las intencionalidades individuales (Giraldo, 2018; Isaacs, 2011; 2016). Esta supone la cooperación de los agentes individuales y un propósito común como condiciones necesarias. Más aún, el que sea colectiva no significa que exista una mente colectiva distinta de las mentes individuales.

En Searle (1992) los estados mentales de los agentes intencionales cuentan con cierta estructura o trasfondo [background] que permiten suponer que tales agentes individuales tienen la intención de cooperar para un propósito común, sin que esto implique que para la adscripción de intencionalidades colectivas debamos conocer la intencionalidad singular de todos y cada uno de los miembros que participan en el grupo, pues el contenido de "nosotros intentamos" ["we-intend"] propio de la

${ }^{6}$ Para Searle (2018) la intencionalidad es un fenómeno biológico común a seres humanos, pero también a otros animales. Sus formas más básicas, biológicamente, son la percepción consciente, el hambre, la sed y emociones como la ira, la lujuria, etc. y, sus formas derivadas, las creencias, deseos, esperanzas. Para él "[t]odos los estados intencionales, sin excepción, son causados por procesos cerebrales y se producen en el cerebro". De momento no hay una explicación biológica satisfactoria, como no lay de la conciencia, de la vida que también son fenómenos biológicos. Para él, el dualismo metafísico ni se contempla. (pp. 44- 45). 
intencionalidad colectiva, no es reductible al contenido de "yo intento" ["I-intend"] propio de la intencionalidad individual, si bien ambas dependen del transfondo [background], i.e., del "conjunto de capacidades mentales no representacionales que permiten que tengan lugar las representaciones", p. 152).

Como ya vimos, Dennett (2000) se refiere además a los sistemas intencionales en sentido amplio, donde incluye las macromoléculas que se duplican así mismas, las plantas, las ratas, los murciélagos, los computadores y, por supuesto, las personas. En todos estos casos interpretamos sus comportamientos "como si [as if] fuera un agente racional que rigiera la 'elección' de sus actos, teniendo en cuenta sus 'creencias' y sus 'deseos'" (p. 40). En el caso de la computadora, Dennett (1985) nos dice que "solo hay paso para llamar a la información poseída creencias de la computadora y a sus metas o submetas deseos" (pp. 10-11). No obstante, aclara que esta noción que propone de sistemas intencionales "no afirma que los sistemas intencionales tengan realmente creencias y deseos, sino que uno puede explicar y predecir su conducta atribuyéndoles creencias y deseos" (pp. 10-11).

En todos estos casos la explicación/predicción intencional sólo se puede llevar a cabo mediante el empleo en ella de términos/conceptos intencionales que son los que en las leyes de las teorías intencionales $\mathrm{T}_{\mathrm{I}}$ aparecen enlazados junto con los demás términos/conceptos no intencionales, en este caso, los términos/conceptos T-no teóricos (de conformidad con el criterio de T-teoricidad de Sneed). Son estas leyes, en las que se relacionan parámetros teóricos intencionales con los parámetros no-teóricos no intencionales con los que caracterizamos los $M_{D}$, las que permiten definir "junto con otras restricciones a las me haremos mención más adelante) los modelos de las teorías intencionales $\mathrm{T}_{\mathrm{I}}$ que no son otros que aquellos sistemas que las satisfacen, i.e., aquellos sistemas en los que los valores concretos de los parámetros empíricos (los datos) y los parámetros teóricos (los intencionales) relacionados en dichas leyes coinciden así sea aproximadamente, como es el caso de teorías de las ciencias sociales que hacen uso de un lenguaje cuantitativo o de magnitudes como es el caso de la economía. Cuando esto ocurre decimos que la $\mathrm{T}_{\mathrm{I}}$ representa adecuadamente el comportamiento de los sistemas que queremos explicar/subsumir. Esto no es otra cosa que la incrustación del $\mathrm{M}_{\mathrm{D}}$ en modelo teórico $\mathrm{M}_{\mathrm{T}}$, siendo " $\mathrm{T}$ " una teoría intencional $\mathrm{T}_{\mathrm{r}}$. Se trata como diría Diez (1998) de una "representación subsuntiva" o "teórica". 


\section{El enfoque intencional en las ciencias sociales}

Este enfoque intencional, diferente del enfoque físico y del enfoque de diseño está presente en casi todas (o quizás en todas) las explicaciones/ predicciones de las ciencias sociales y constituyen su rasgo distintivo e idiosincrásico, sin con ello desconocer que existan otras diferencias como las que se refieren a la naturaleza abierta o cerrada de sus sistemas, la posibilidad o no de aislar y repetir los experimentos y, en general, todas aquellos tópicos a los que Popper (1957) hace referencia en sus críticas a la doctrina historicista.

Las $\mathrm{T}_{\mathrm{I}}$ tienen como dominio, universo o clase de referencia sistemas intencionales cuyo comportamiento solo se puede explicar/predecir apelando a términos/conceptos intencionales propios del lenguaje intencional $\mathrm{L}_{\mathrm{r}}$. De ese modo, si queremos entender o controlar su comportamiento, debemos -como dice Sneed (2011)- imputarle a los individuos o agentes intencionales de los sistemas intencionales, atributos o predicados intencionales (en adelante $\mathrm{P}_{\mathrm{I}}$ ), aunque éstos, "no son parte del comportamiento a ser explicado" (Sneed, 2011, p. 14), algo en lo que coincide con Dennett.

E1 $L_{I}$ es distinto del lenguaje descriptivo (en adelante $L_{D}$ ). Este último está constituido por términos/conceptos descriptivos o T-no teóricos (si nos atenemos al criterio de T-teoricidad de Sneed) y es que empleamos para describir el comportamiento de algunos individuos seres humanos, organismos vivos, artefactos complejos, etc.- que, como observadores "externos", queremos explicar/predecir e incluso controlar. Dicho comportamiento hacen parte de lo que en el EM se conoce como "aplicaciones pretendidas" o "intencionales" I, cuyos datos, recopilados mediante observación, medición y experimentación, podemos estructurar o sistematizar bajo la forma de modelos de datos [models of data] (en adelante MD). Tantos los I como los MD son subconjuntos de los modelos potenciales parciales $\mathrm{M}_{\mathrm{pp}}$, i.e, de aquellos modelos que resultan de recortar (mediante la función recorte $\mathrm{r}$ ) los términos/conceptosT-teóricos en los modelos potenciales $\mathrm{M}_{\mathrm{p}}$ que constituyen el aparato conceptual básico de la teoría. Formalmente: $r: \mathrm{M}_{\mathrm{p}} \mathrm{M}_{\mathrm{pp}}$.

Para poder establecer qué datos corresponden a qué sistema real, se hace necesario coordinar esas estructuras D con los sistemas I del que fueron recolectados, lo que implica introducir una función que llamaremos " $\Psi$ " que garantice tal coordinación. Formalmente: $\Psi$ : D I.

Con la introducción de los términos/conceptos intencionales teóricos en $\operatorname{los} \mathrm{M}_{\mathrm{pp}}\left(\mathrm{T}_{\mathrm{I}}\right)$ de los que los que las aplicaciones pretendidas $\mathrm{I}\left(\mathrm{T}_{\mathrm{I}}\right)$ son un subconjunto $\left(\mathrm{I}\left(\mathrm{T}_{\mathrm{I}}\right) \mathrm{M}_{\mathrm{pp}}\left(\mathrm{T}_{\mathrm{I}}\right)\right)$, los $\mathrm{M}_{\mathrm{pp}}\left(\mathrm{T}_{\mathrm{I}}\right)$ se convierten en modelos potenciales $\mathrm{M}_{\mathrm{p}}\left(\mathrm{T}_{\mathrm{I}}\right)$, de tal modo que de ellos, así enriquecidos con términos $\mathrm{T}_{\mathrm{I}}$-teóricos, 
cobraría sentido preguntarse si son modelos actuales o efectivos $\mathrm{M}\left(\mathrm{T}_{\mathrm{I}}\right)$, i.e., si encajan en o pueden ser subsumidos por el núcleo formal $\mathrm{K}\left(\mathrm{T}_{\mathrm{T}}\right)$, vale decir, si satisfacen las constricciones que establece $\mathrm{T}_{\mathrm{I}}$ : sus leyes, sus condiciones de ligadura [constraints] como "principios-puente" que conectan los distintos modelos de una misma teoría y los vínculos interteóricos [links] que conectan dichos modelos con los modelos de teorías distintas, tal y como lo establece la aserción empírica o enunciado Ramsey-Sneed que acompaña a las distintas teorías. Formalmente, lo que en este caso la aserción empírica dice es que I $\operatorname{Con}\left(\mathrm{K}\left(\mathrm{T}_{\mathrm{I}}\right)\right.$. El $\operatorname{Con}\left(\mathrm{K}\left(\mathrm{T}_{\mathrm{I}}\right)={ }_{\text {def }}: \operatorname{Po}\left(\mathrm{M}\left(\mathrm{T}_{\mathrm{I}}\right)\right) \mathrm{GC}\left(\mathrm{T}_{\mathrm{I}}\right) \operatorname{Po}\left(\mathrm{GL}\left(\mathrm{T}_{\mathrm{I}}\right)\right)\right.$, siendo "Po(M(T $\left.\mathrm{T}_{\mathrm{I}}\right)$ ")" conjunto potencia de modelos de $\mathrm{T}_{\mathrm{i}}$, "GC (Ti)" el conjunto de condiciones de ligadura o [contraints] de Ti y "Po(GL(Ti)" el conjunto potencia de vínculos interteóricos [links] globales.

Lo que la aserción empírica (enunciado Ramsey-Sneed) nos dice es que ciertos sistemas físicos, descritos mediante términos/conceptos $T_{\mathrm{I}}$ no teóricos $\left(\operatorname{los} \mathrm{M}_{\mathrm{D}}\left(\mathrm{T}_{\mathrm{I}}\right)\right.$, pueden ser enriquecidos o ampliados mediante términos/conceptos $\mathrm{T}_{\mathrm{I}}$-teóricos y si así enriquecidos satisfacen las leyes, las condiciones de ligadura y los vínculos interteóricos que impone la teoría intencional $\mathrm{T}_{\mathrm{I}}$ entonces podemos decir que son modelos $\mathrm{M}\left(\mathrm{T}_{\mathrm{I}}\right)$ o, lo que es equivalente, que son subsumibles o encajan en $\mathrm{T}_{\mathrm{I}}$ (Forge, 2002).

Pero dado que las teorías maduras son una red teórica, i.e., un conjunto de elementos teóricos ordenados parcialmente por relaciones de especialización, la subsunción modelo-teórica de los sistemas intencionales por parte de $\mathrm{T}_{\mathrm{I}}$, no solo incluye la(s) ley(es fundamental(es) de $\mathrm{T}_{\mathrm{I}}$ (Fig. 1), sino también leyes especiales cuando está involucrado un pedazo de la red teórica. Estas leyes, especiales, junto con ligaduras [constraints] especiales y con los vínculos interteóricos especiales constituyen las constricciones especiales que los sistemas reales ampliados deben cumplir para ser modelos reales $\mathrm{M}\left(\mathrm{T}_{\mathrm{I}}\right)$ (Ver Fig. 2). 


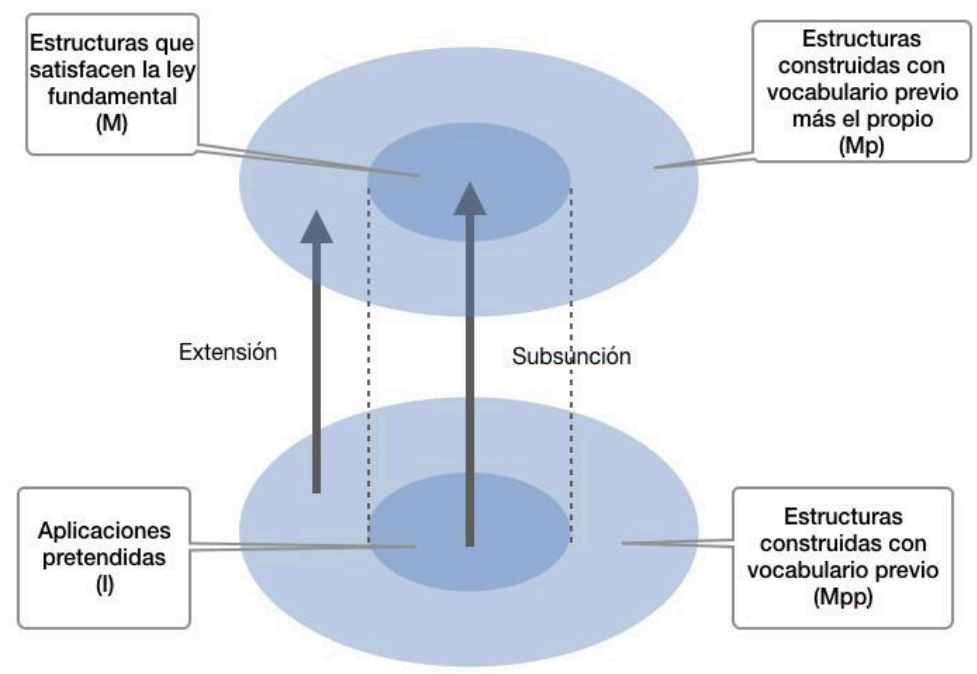

Figura 1. Modelo de explicación por subsunción modelo-teórica en un solo elemento teórico

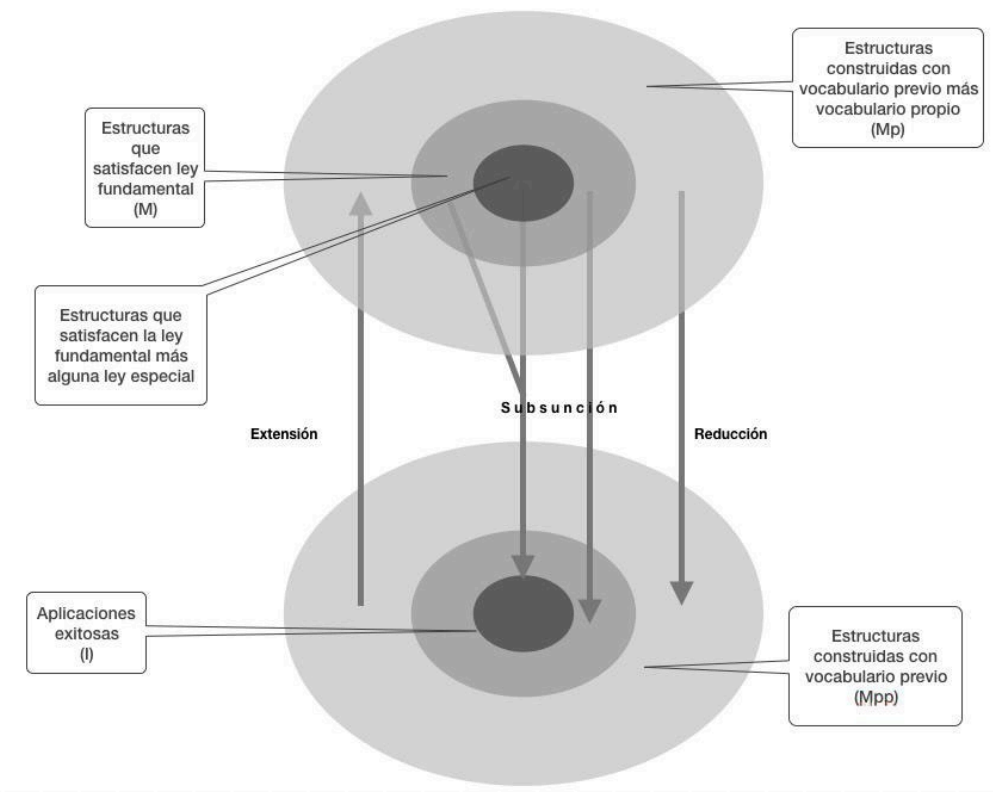

Figura 2. Modelo de explicación modelo-teórico en una red teórica 
En algunas ocasiones términos/conceptos $\mathrm{T}_{\mathrm{I}}$-teóricos de que nos valemos para explicar/predecir los distintos sistemas sociales son causas del comportamiento de esos sistemas que queremos explicar/predecir. Tal es el caso de la Teoría de elección racional TER en las ciencias sociales donde las razones (creencias o deseos) son causas de la elección.

Sin la adscripción de términos/conceptos intencionales a los explananda de las ciencias sociales o, al menos en la mayoría de éstas, no es posible explicar/predecir nada respecto de la conducta de los agentes. El conductismo en psicología, las descripciones etnográficas en antropología y en buena parte de las investigaciones cualitativas, realizan descripciones en las que se hacen uso exclusivo de un vocabulario descriptivo $\mathrm{T}_{\mathrm{I}}$-no teórico y sin bien poseen un rico contenido informacional, tienen un nulo poder explicativo/ predictivo. Refiriéndose al caso específico del conductismo de Skinner, Dennett (1985) afirma que lo que se busca es

tratar encuadrar las predicciones exclusivamente en lenguaje no intencional $\left[\mathrm{L}_{\mathrm{NI}}\right]$, prediciendo respuestas corporales a los estímulos físicos, pero hasta el momento esto no le ha proporcionado el deseado modo alterno de predicción y explicación, como acaso puede indicarlo un análisis extremadamente sucinto (p. 20).

\section{Y más adelante agrega:}

El diseño experimental de Skinner supone eliminar lo intencional, pero lo que hace es meramente enmascararlo. Las predicciones no intencionales de Skinner funcionan hasta donde lo hacen, no porque Skinner haya encontrado en verdad leyes conductuales no intencionales altamente confiables que subyacen en sus situaciones experimentales (la rata desea comida y cree que conseguirá comida al presionar la barra - algo para lo cual se le ha dado buena evidencia - así que presiona la palanca) [...] el éxito aparente del conductismo skinneriano descansa en las predicciones intencionales ocultas. (p. 23. Las cursivas son mías).

Las "regularidades" de superficie observadas por Skinner en sus experimentos de laboratorio, enmascaran regularidades intencionales profundas, que es lo que el uso exclusivo de un lenguaje puramente descriptivo -como lo es el lenguaje skinneriano- no permiten avizorar. Para Dennett, Skinner

está en lo correcto al buscar regularidades mecanísticas en las actividades de sus sujetos, pero no hay razón para suponer que tales regularidades se 
mostrarán en la superficie, en la conducta crasa -excepto, como hemos visto, cuando ponemos una camisa de fuerza artificial a una regularidad intencional (p. 24).

El carácter intencional de los sistemas intencionales ha sido objeto de amplias discusiones. Quine -seguidor de la psicología skinneriana- defiende un punto de vista extensionalista y rechazan cualquier enfoque intensional como "s" (Quine, 1960). Aunque la intencionalidad como "c" no es equivalente a la intensionalidad como "s", pues la primera es una propiedad de la mente (cerebro) y la segunda una propiedad de las proposiciones cuando estas responden a ciertos requisitos lógicos -la no sustitución de sus términos por términos co-extensivos salva veritatae- todas los enunciados o proposiciones intensionales con "s" son intencionales con " $\mathrm{c}$ ", aunque la conversa no siempre se cumple.

M. Bunge (1996), pese sus críticas a la metodología individualista en las ciencias sociales, reconoce el importante papel que en ellas desempeña la intencionalidad:

Los conceptos de elección, decisión y acción racionales se encuentran en el centro de un gran número de teorías, amplias o estrechas, en todas las ciencias sociales: las teorías de las utilidades, de las decisiones y de los juegos, así como en la microeconomía neoclásica" (Bunge, p. 495. La cursiva es mía).

\section{Presentación de algunos casos de teorías de las ciencias sociales como teorías intencionales}

Hasta ahora hemos afirmado que si no todas, al menos la inmensa mayoría de las teorías de las ciencias sociales que han sido formalmente reconstruidas son teorías intencionales $\mathrm{T}_{\mathrm{I}}$. Dentro del conjunto amplio de $\mathrm{T}_{\mathrm{I}}$ quiero destacar tres que me parecen las más relevantes en las ciencias sociales, en particular, en la economía que, sin lugar a dudas, es (junto con la lingüística) la disciplina que en las ciencias sociales que más desarrollos ha logrado.

Esas teorías son (i) la Teoría de la elección racional (en adelante TER), sin lugar a dudas la $\mathrm{T}_{\mathrm{I}}$ más general; (ii) la Teoría de decisiones (en adelante TD) que se ocupa de la toma de decisiones cuando existe una incertidumbre asociada a algunas alternativas y, (iii) la Teoría de juegos (en adelante TJ) que es una extensión de la TD cuando la incertidumbre viene dada por las 
acciones de otros decisores, algo que no ocurre en la TD (Ferreira, 2009, pp. 76-77) 7 .

La TER busca dar cuenta de las creencias, que, como razones (causas) llevan a un agente a actuar. Este intencionalmente elige aquella acción que, cree, le permite el mejor resultado, entre un conjunto amplio de resultados posibles. Mediante la función preferencia, el agente jerarquiza los resultados posibles de las distintas acciones, asignándoles probabilidades, para de ese modo elegir la opción más óptima para lograr el equilibrio. Cuando esto ocurre decimos que la acción del agente es una acción racional.

La teoría de decisiones TD y la Teoría de juegos TJ son especializaciones de la TER.

En An Architectonic (1987) se presenta una reconstrucción conjuntista informal (parcial) de la TD en la versión de Jeffrey (1965) en la que se advierte que se trata de una teoría que "pertenece claramente a las ciencias de la conducta" y que "[s]olo tiene sentido tratar de aplicarla a los seres humanos o, a lo sumo, a los organismos desarrollados y a las máquinas" (p. 74), i.e., a sistemas intencionales en sentido amplio.

La teoría de juegos TJ, en particular, la Teoría de juegos finitos en forma extensiva (TEG por sus siglas en inglés), con un número finito de jugadores y de movimientos fue reconstruida en términos del EM por García de la Sienra y Reyes (2000).

Si bien en la TER se trata de una acción intencional, racional y optimizadora, en el caso de la TD, el agente decisor supone-quizás erróneamente- que el medio y los otros agentes son constantes y que la única variable interviniente son las decisiones del agente decisor. En la $\mathrm{TJ}$, en cambio, el agente decisor no es la única variable interviniente y su decisión en situación de incertidumbre tiene en cuenta los demás agentes decisores a quienes aquel también considera racionales. La TJ en particular, la Teoría de juegos finitos en forma extensiva (TEG por sus siglas en inglés), con un número finito de jugadores y de movimientos fue reconstruida en términos del EM por A. García de la Sienra y P. Reyes (2000). Asó como para estos dos autores la teoría neoclásica del equilibrio general es una especialización de la TJ, para Ferreira (2009) la TJ es una extensión de la TD para "aquellas situaciones en las que la incertidumbre vienen dada por las acciones de otros decididores" (p. 76). No obstante, hay que señalar que la TD y la TJ son distintas y la lógica que les subyace es también distinta.

${ }^{7}$ La teoría de juegos TJ, en particular, la Teoría de juegos finitos en forma extensiva (TEG por sus siglas en inglés), con un número finito de jugadores y de movimientos fue reconstruida en términos del EM por A. García de la Sienra y P. Reyes (2000). 
De cualquier modo, todas son intencionales. En el primer caso, García de la Sienra y Reyes (2000) afirman:

la economía neoclásica es una especialización de la teoría de juegos, aplicada cuando las estrategias, o más bien las acciones de otros jugadores, no puede afectar la situación dada por "natural", pero todos los jugadores deben reaccionar en una situación tratando de maximizar sus utilidades dentro de las restricciones impuestas por la misma situación (esta situación es usualmente un sistema de precios, y es por eso que a los jugadores se les llama "tomadores de precios" ["price takers"] (p. 66).

En estas teorías -como lo advierten muchos autores- muchas de las decisiones del agente dependen de su juicio, vale decir de su capacidad para evaluar y sintetizar la información que considere pertinente en una determinada elección. Tanto la TD como en la TJ lo que se hace es conectar las utilidades esperadas del agente con sus juicios de probabilidad subjetiva ${ }^{8}$ y aunque es imposible atribuirle a los jugadores una racionalidad perfecta, lo que la TJ plantea es una tendencia hacia el equilibrio. En ella la expectativa (del agente) y la probabilidad (subjetiva) no pueden ser medidas independientes de la teoría. Si esto es así, estas dos funciones son TJ-teóricas y desempeñan un papel fundamental en la explicación/predicción científica.

Una de las TJ más exitosas es la Teoría del equilibrio general del Nash, galardonada con el premio Nobel de Economía en 1994 que, a diferencia de Teorías de juego como las de von Neumann y Morgenstern que le precedieron, no es una TJ de suma cero, donde lo que gana un jugador lo pierden los demás. En ella cada jugador sabe que el otro -aunque pueden ser varios- también es racional y, en consecuencia, adopta la mejor estrategia para conseguir el equilibrio, i.e., el mejor resultado para cada uno ellos considerado individualmente. Esta teoría ha tenido numerosas aplicaciones, no solo en economía, sino en sociología, en psicología, en derecho, en política, en la resolución de conflictos, en ecología, etc. Una variante de ella es el "dilema del prisionero" donde la cooperación maximiza la ganancia.

Otro caso interesante de $\mathrm{T}_{\mathrm{I}}$ que quiero destacar es el de la Teoría de las emociones de W. Wundt, reconstruida con la metodología del EM por R Reisenzein (2000). Wundt, considerado uno de los fundadores de la psicología, se propone explicar la naturaleza y función de las emociones

${ }^{8}$ Se pueden distinguir tres tipos de probabilidad: lógica, subjetiva y objetiva. La primera considera que los enunciados de probabilidad son acerca de las relaciones lógicas (inductivas) de la probabilidad; la segunda se refiere al grado de creencia (confianza) razonable de un agente de que ocurra un evento $y$, la tercera, tiene que ver con el índice de frecuencia relativa de la ocurrencia de un evento una de cuyas versiones es la teoría de las propensiones. 
humanas como resultado de la fusión de dos o más sensaciones [feeling] irreductibles entre sí, entre las que destaca las de placer o displacer, disgusto, excitación, inhibición o tranquilización, tensión y relajación. En contra de lo piensa Brentano, para él estas no son estados mentales intencionales, pues no tienen como correlato objeto alguno. Son el efecto de estados mentales que sí son intencionales. Francisca está disgustada por haber perdido el tren, pero su disgusto es causado por el conocimiento de haber perdido el tren. De este modo, su sensación de disgusto es causada por un estado mental: el conocimiento de haber perdido el tren (Reisenzein, 2000, pp. 220-221). Esta reflexión resulta importante al momento de explicar/predecir la conducta de los individuos apelando a estados mentales como las creencias, los deseos, las preferencias, los conocimientos, etc. qua estados intencionales.

Para finalizar, es preciso enfatizar que en la TER como en la TJ, sus análisis parten de un individuo que se supone es completamente racional y que, además, posee una información completa y un perfecto ordenador interno; un individuo que, como racional, "escoge la acción que él mismo, con base en una distribución de probabilidades, calcula de un modo y que instrumentalmente es la mejor para satisfacer sus preferencias" (Hollis, 1998, p. 129). Hollis lo ilustra con el caso de un Crusoe que en su isla desierta decide si vale la pena tejer una red para pescar un mayor número de peces o si lo hace con un palo con una punta afilada. Tejer la red no solo implica que probablemente mañana se puedan atrapar más peces, sino el esfuerzo de tejerla, pero al mismo tiempo, dejar de atrapar peces mientras la teje. Crusoe, como la TD o la TJ, cuenta con una distribución subjetiva de probabilidades, completa y consistente, y por tanto, dispone de un juicio para calcular la utilidad esperada, lo que implica comparar la probabilidad de pesca tejiendo la red o dejándola de tejer. Esto -como se ve claro en la en las dos teorías anteriores- tiene un tratamiento matemático, probabilista, y es mediante él como se puede establecer la máxima utilidad esperada o realización posible al finalizar del juego. En palabras de Hollis (1998):

Actuamos racionalmente cuando sabemos qué es lo que queremos, tenemos la astuta idea de saber cuán plausible es cada curso de acción para satisfacernos y a qué coste, y escogemos la acción que, en consecuencia, nos parezca el medio más eficaz para satisfacerla [...]. Los agentes racionales, pueden tener cualquier (consistente) preferencia si y solo si sus elecciones maximizan correspondientemente la utilidad esperada que acarician (p. 130). 
Sin embargo, hasta ahora hemos hablado preferencialmente del homo economicus donde la decisión estratégica del agente depende de la elección y decisiones racionales con base en un cálculo estratégico maximizador, cuando el agente ha identificado las utilidades que tal cálculo entraña. De este modo, el homo economicus sería para Hollis (1998):

un individuo harto mecánico, un mero intermediario entre unas preferencias dadas y una elección racional automáticamente computada. Es el caso del tipo-ideal, donde los agentes son absolutamente racionales y todos saben que lo son [y donde] el carácter psicológico no interviene para nada (p. 304).

La pregunta entonces es la de cómo se relaciona una teoría tan abstracta con el mundo real y, sobre todo, con el cúmulo las elecciones que hacemos a diario. La respuesta que propone Hollis es que hay que combinar ese homo economicus individualista que busca maximizar sus utilidades, con un homo sociologicus que se desempeña en un contexto social, i.e., con una persona socializada y socialmente ubicada, de modo que su elección - como acción instrumentalmente racional- se da en un contexto social que, si bien no determina su elección, al menos sí la condiciona. Por todo esto concluye Hollis:

Insistir en explorar el mundo social desde dentro no es conceder el triunfo a la comprensión [Verstehen], puesto que la explicación [Erklärung] aún puede tener la última palabra. Pero tendrá que ser la última palabra. Los primeros pensamientos sobre las normas, las instituciones y las prácticas son tan contrarios a la teoría sistémica como la teoría de juegos. Tales "estructuras", aun si externas a cada uno de los actores, son colectivamente internas a todos por estar entretejidas con redes públicas y significados que no se dan en las colonias de termitas ni en los sistemas planetarios (p. 198).

\section{A modo de conclusión}

En síntesis, dada la importancia de la intencionalidad y del $\mathrm{L}_{\mathrm{I}}$ en las ciencias sociales, se hace necesario emprender una rigurosa investigación en torno a la estructura de las $\mathrm{T}_{\mathrm{I}}$ que, complementada con trabajo realizado por Sneed (2011) sobre la estructura sintáctico/semántica del lenguaje intencional $\mathrm{L}_{\mathrm{I}} \mathrm{y}$ de su diferencia con el lenguaje descriptivo $\mathrm{L}_{\mathrm{D}}$, permita dar cuenta de las diferencias y similitudes entre las ciencias sociales y las naturales, sin excluir de las primeras la explicación/predicción de sus 
explananda. En aquellas los lenguajes intencionales resultan fundamentales para los procesos de explicación/predicción de su conducta y sus leyes deben incluir términos/conceptos intencionales, pues sin ellos no es posible explicar/predecir las diferentes conductas de los actores sociales si no en todas las sociales, al menos sí en su mayoría.

\section{Referencias bibliográficas}

Bunge, M (1996). Debate sobre las ciencias sociales. Buenos Aires, Argentina: Sudamericana.

Davidson, D. (1980). Essays on Actions and Events. Oxford, Inglaterra: Clarendon Press.

Dennett, D.C. (1985). Cuadernos de crítica. Sistemas intencionales. Ciudad de México, México: Universidad Nacional Autónoma de México.

Dennett, D.C. (2000). Tipos de Mentes. Hacia una comprensión de la conciencia. Barcelona, España: Debate.

Díez, J. (1998). Hacia una teoría general de la representación científica. Theoria, 13(1), 113-139.

Elster, J. (1995). Ulises y las sirenas. Estudio sobre racionalidad e irracionalidad. Ciudad de México, México: Fondo de Cultura Económica.

Ferreira, J.L. (2009). La teoría de juegos, sus aplicaciones en economía y su metodología. En J. C. García-Bermejo (Ed.), Sobre la Economía y sus métodos. (pp. 73-88). Madrid, España: Editorial Trotta.

Forge, J. (2002). Reflections on Structuralism and Scientific Explanation. Synthese, 130(1), 109-121.

García de la Sienra, A. y Reyes, P. (2000). The Theory of Finite Games in Extensive Form. En W. Balzer, J. D. Sneed y C. U. Moulines (Eds.), The Structuralist Knowlwdge Representation. Paradigmatic Examples. Pp. 51-67. Amsterdam, Países Bajos: Radopi.

Giraldo, L. I. (2018). Intencionalidad y acciones colectivas. Una lectura desde Searle e Isaacs. Bogotá, Colombia: Universidad del Rosario.

Gonzalez, L. (2016). El problema de la teleología y la metáfora del diseño en biología: cuestiones epistemológicas e implicaciones didácticas. Tecné Episteme Y Didaxis: TED, (40), 149-173. doi: 10.17227/01203916.6151.

Hempel, C. G. y; Oppenheim, P. (1948). Studies in logic of explanation. Philosophy of Science, 15(2), 135-175.

Hollis, M. (1998). Filosofia de las ciencias sociales. Una introducción. Barcelona, España: Ariel.

Isaacs, T. (2011). Moral Responsability in Collective Contexts. Nueva York, EUA: Oxford University Press.

Isaacs, T. (2016). Collective Moral Responsibility and Collective Intention. Midwest

Studies in Philosophy, 30(1), 59-73. doi: 10.1111/j.1475-4975.2006.00128.x. Jeffrey, R. (1965). The Logic of Decision. Nueva York, EUA: McGraw-Hill. Kuhn, T.S. (2002). El camino desde la estructura. Barcelona, España: Paidós. 
Mayr, E. (2006). Por qué es única la biología. Consideraciones sobre la autonomía de una disciplina cientifica. Buenos Aires, Argentina: Katz.

Moulines, C.U. (2007). Explicación teórica y compromisos ontológicos. Quderns de filosofía i ciencia, (37), 7-14.

Nagel, E. (1991). La estructura de la ciencia. Barcelona, España: Paidós.

Popper, K.R. (1957). The Poverty of Historicism. Nueva York, EUA: Routledge. Quine, W. (1960). Word and Object. Nueva York, EUA: Wiley.

Reisenzein, R. (2000). Wundt's Three Dimensional Theory of Emotion. En W. Balzer, J. D. Sneed y C. U. Moulines (Eds.), Structuralist Knowledge Representation. Paradigmatic Examples (pp. 219-250). Amsterdam, Países Bajos: Rodopi.

Ruse, M. (2000). Teleology: Yesterday, today, and tomorrow? Studies in History and Philosophy of Biological \& Biomedical Sciences, 31(1), 213-232. doi: 10.1016/ S1369-8486(99)00046-1.

Searle, J.D. (1980). Minds, Brains, and Programs. The behavioral and brain sciences, 3(3), 417-424. doi: 10.1017/S0140525X00005756.

Searle, J.D. (1983). Intentionality. An Essay in the Philosophy of Mind. Cambridge, Inglaterra: Cambridge University Press.

Searle, J.D. (1990). Collective Intentions and Actions. En P. Cohen, J. Morgan y M. E. Pollock (Eds.), Intentions in Comunication (pp. 401-415). Cambridge, Inglaterra: Cambridge University Press.

Searle, J.D. (1992). Intencionalidad. Un ensayo en filosofía de la mente. Madrid, España: Tecnos.

Searle, J.D. (1995). The Construction of Social Reality. Nueva York, EUA: The Free Press.

Searle, J.D. (2001). Rationality and Action. Cambridge, EUA: The MIT Press.

Searle, J.D. (2010). Making the Social World. Nueva York, EUA: Oxford University Press.

Searle, J.D. (2018). Ver las cosas tal como son. Madrid, España: Cátedra.

Sneed, J.D. (2011). Notes on Intensional Theories. Discusiones Filosóficas, (18), 13-49.

von Wright, G. H. (1979). Explicación y comprensión. Madrid, España: Alianza Editorial. 
